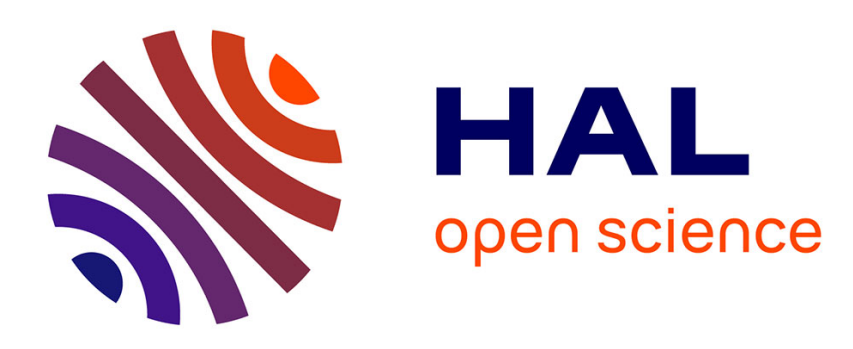

\title{
Necessary and Sufficient Conditions on the Exponential Stability of Positive Hyperbolic Systems
}

Liguo Zhang, Christophe Prieur

\section{To cite this version:}

Liguo Zhang, Christophe Prieur. Necessary and Sufficient Conditions on the Exponential Stability of Positive Hyperbolic Systems. IEEE Transactions on Automatic Control, 2017, 62 (7), pp.3610-3617. 10.1109/TAC.2017.2661966 . hal-01385745v2

\section{HAL Id: hal-01385745 \\ https://hal.science/hal-01385745v2}

Submitted on 20 Jan 2017

HAL is a multi-disciplinary open access archive for the deposit and dissemination of scientific research documents, whether they are published or not. The documents may come from teaching and research institutions in France or abroad, or from public or private research centers.
L'archive ouverte pluridisciplinaire HAL, est destinée au dépôt et à la diffusion de documents scientifiques de niveau recherche, publiés ou non, émanant des établissements d'enseignement et de recherche français ou étrangers, des laboratoires publics ou privés. 


\title{
Necessary and Sufficient Conditions on the Exponential Stability of Positive Hyperbolic Systems (full version)
}

\author{
Liguo Zhang and Christophe Prieur
}

\begin{abstract}
In this paper, a strict linear Lyapunov function is developed in order to investigate the exponential stability of a linear hyperbolic partial differential equation with positive boundary conditions. Based on the method of characteristics, some properties of the positive solutions are derived for the hyperbolic initial boundary value problems. The dissipative boundary condition in terms of linear inequalities is proven to be not only sufficient but also necessary under an extra assumption on the velocities of the hyperbolic systems. An application to control of the freeway traffic modeled by the Aw-Rascle traffic flow equation illustrates and motivates the theoretical results. The boundary control strategies are designed by integrating the on-ramp metering with the mainline speed limit. Finally, the proposed feedback laws are tested under simulation, first in the free-flow case and then in the congestion mode, which show adequate performance to stabilize the local freeway traffic.
\end{abstract}

Index Terms-Positive hyperbolic systems, Lyapunov function, distributed parameter systems, exponential stability, Aw-Rascle equations.

\section{INTRODUCTION}

Many physical systems having an engineering interest are represented by a system of one-dimensional hyperbolic partial differential equations (PDEs). Typical examples include the telegrapher equations for electrical circuits with transmission lines [25], the shallow water equations for open channels [10], [7], and the Aw-Rascle traffic flow model for road traffic systems [2].

The problem of analyzing exponential stability of the equilibrium for linear or quasilinear hyperbolic systems has been considered in the literature for more than 30 years. One main contribution of [17] is the trajectory-based technique via direct estimation of the solutions and their derivatives along the characteristic curves. Recent results have been extended to the case of conservation laws with perturbation source terms [22], [23], and absolutely exponential stability for switched hyperbolic systems [1], [21] under suitable dissipative boundary conditions.

On the other hand, a Lyapunov stability analysis technique is proposed in [6], with a strict quadratic Lyapunov function to

This work is partially supported by the National Natural Science Foundation of China (NSFC, grant No. 61374076, No. 61533002) and the International Cooperation and Exchange Program of NSFC (grant No. 61111130119).

L. Zhang is with School of Electronic Information and Control Engineering, Beijing University of Technology, and with Key Laboratory of Computational Intelligence and Intelligent Systems, Beijing, 100124, China (e-mail: zhangliguo@bjut.edu.cn).

C. Prieur is with Univ. Grenoble Alpes, CNRS, Gipsa-lab, F-38000 Grenoble, France (e-mail: christophe.prieur@gipsa-lab.fr). analyze the exponential stability of the equilibrium of conservation laws. The sufficient dissipative boundary condition is known to be strictly weaker than the one of [17] and to allow for numerically tractable methods for the design of stabilizing boundary controls [8] However, the approach by a quadratic Lyapunov function is not always effective to prove stability for hyperbolic systems. It has been shown in [3] that there exist stable $2 \times 2$ linear hyperbolic systems for which there does not exist any quadratic Lyapunov function.

In this paper, we consider the case in which hyperbolic systems belong to a class of positive systems whose state variables remain non-negative whenever initialized in the positive conditions. The positivity constraint on system states is motivated by the engineering physical nature of the variables involved (pressure, mass flux, density, vehicle speed and so on). The stability and other properties of positive systems often present some peculiar features [14], [15], [18], [24], which simplify the system analysis.

A necessary and sufficient condition for exponential stability of positive linear hyperbolic systems is presented by means of a novel strict Lyapunov function. To the best of our knowledge, this is the first work dealing with such a converse explicit Lyapunov result. Note that [5, Proposition 3.7] implies that the dissipative boundary condition is a necessary and sufficient condition for stability of linear hyperbolic systems with dimension 1 to 5 . Our necessary discussion relies on the assumption that velocities of hyperbolic systems have a common factor. Then, we could find a common time sample at which the dynamics of all states are projected to the boundaries; it transforms the dynamic of boundary conditions into a time-delay difference equation. The obtained conditions are written in terms of linear inequalities which can be solved numerically. The context of a continuous selection of boundary conditions in an interval matrix is also considered, supposing each entry belongs to a certain interval of the real line. Absolutely exponential stability also holds if and only if the upper bound satisfies the dissipative conditions.

The motivating application for the theoretical results presented in this paper is the development of dissipative boundary conditions with a strict linear Lyapunov function to regulate traffic states (vehicle density, speed) in a freeway link. Adopting boundary control strategies are particularly natural and necessary for freeway traffic systems, since the available control devices often depend on traffic signals for on-ramp metering and VMSs (Variable Message Signs) for regulating vehicle speed usually located at boundaries or cross-sections 
of the freeway link. Inside the freeway section, the number of vehicles is conservative. The dissipative boundary condition obtained from our theoretical contribution allows us to design the stabilizing feedback laws by integrating the on-ramp metering with the speed limiting control in the distributed control action.

The problem of freeway traffic control has been considered for a long time in the literature as reported in the survey [20] paper which involves a comprehensive bibliography.

In this paper, we go a step further since the control design is derived and analyzed directly from the linearized Aw-Rascle partial differential equations without any model approximation and discretisation. As Aw-Rascle traffic flow model, the speed dynamics might propagate upstream or downstream along the freeway for the same flow-rate of traffic, in practice, also called in the free-flow traffic or in the congestion mode. For the application, two different boundary feedback strategies are proposed in this paper by taking advantage of the traffic measurements coming from the upstream or downstream, respectively. In details, as the steady states in the free-flow mode, we detect downstream traffic states and integrate ramp metering with variable speed limit to control the upstream boundary of the freeway link, while in the congestion mode, the control design is the inverse. The theoretical contributions guarantee the exponential convergence of the system when closing the loop with the feedback control.

This paper is organized as follows. The class of positive linear hyperbolic systems is given in Section II. Wellposedness and positive properties of solutions of positive linear hyperbolic system are also discussed. In Section III, our main results on the necessary and sufficient conditions are derived for the exponential stability of positive linear hyperbolic systems. Finally, in Section IV, as a matter of illustration, an application of boundary feedback control of freeway traffic based on the linearized Aw-Rascle traffic flow equations is presented.

Notation. The set of non-negative integers and reals is $\mathbb{N}$ or $\mathbb{R}_{+}$, respectively. $\mathbb{R}_{+}^{n \times n}$ or $\mathbb{R}_{+}^{n}$ is the set of $n$-order square non-negative matrices or vectors. A matrix (in particular, a vector) $A$ with entries in $\mathbb{R}_{+}$is called a non-negative matrix (vector), and it is denoted as $A \succeq 0$. It is said to be positive ( $A \succ 0$ ), if all its entries are positive. The expression $A \succeq B$, indicate the difference $A-B$ is non-negative. The term non-positive, negative are defined analogously as $\preceq$ and $\prec$. $\operatorname{row}_{i}(A)$ denotes the $i$ th row of matrix $A$. A real symmetric matrix $A$ is positive definite (resp. semi-definite positive), if all its eigenvalues are positive (resp. non-negative); in that case we will use the respective notation $A>0$ and $A \geq 0$. Given a function $g$ : $[0,1] \rightarrow \mathbb{R}^{n}$, we define its $L^{1}$-norm as $\|g\|_{L^{1}(0,1)}=\int_{0}^{1}\|g(x)\| d x$, where $\|\cdot\|$ is the Euclidian norm in $\mathbb{R}^{n}$. We call $L^{1}(0,1)$ the space of all measurable functions $g$ for which $\|g\|_{L^{1}(0,1)}<\infty$.

\section{Positive Hyperbolic Systems: Mathematical PRELIMINARIES}

Consider a class of positive linear hyperbolic systems described by the following equation:

$$
\partial_{t} \xi(x, t)+\Lambda \partial_{x} \xi(x, t)=0, \quad t \in \mathbb{R}_{+}, \quad x \in[0,1],
$$

where $\xi:[0,1] \times \mathbb{R}_{+} \rightarrow \mathbb{R}^{n}$. Assume $\Lambda \in \mathbb{R}_{+}^{n \times n}$ is a diagonal positive definite matrix such that $\Lambda=\operatorname{diag}\left\{\lambda_{1}, \lambda_{2}, \ldots, \lambda_{n}\right\}$, with $\lambda_{i}>0$, for all $i \in\{1, \ldots, n\}$. The boundary condition is written as

$$
\xi(0, t)=G \xi(1, t), \quad t \in \mathbb{R}_{+},
$$

where matrix $G$ belongs to $\mathbb{R}_{+}^{n \times n}$. We shall consider the initial condition given by

$$
\xi(x, 0)=\xi^{0}(x), \quad x \in[0,1],
$$

for a given function $\xi^{0}:[0,1] \rightarrow \mathbb{R}^{n}$.

The linear hyperbolic system (1)-(3) is called positive if the trajectories of the system starting from any non-negative initial conditions remains forever non-negative. As example $\xi$ could be related to the gas flow in pipelines as considered in [13]. It could also coupled wave equations for dynamics of stimulated Raman scattering. See in also [11] where the positivity of the solutions is assumed.

The existence of the solution of the hyperbolic system (1)-(3) in the set $C^{0}\left([0, \infty), H^{1}(0,1)\right) \cap C^{1}\left([0, \infty), L^{2}(0,1)\right)$ holds for initial condition $\xi^{0}$ in $H^{1}(0,1)$ satisfying the zeroorder compatibility condition $\xi^{0}(0)=G \xi^{0}(1)$ (see e.g. [5] or [4, Theorem A.1]).

In the context of positive hyperbolic systems, we are able to state the following:

Proposition 1: Assume the matrix $G \in \mathbb{R}_{+}^{n \times n}$ in the boundary condition (2) is non-negative, i.e., $G \succeq 0$. Then, for any function $\xi^{0}:[0,1] \rightarrow \mathbb{R}^{n}$ in $H^{1}(0,1)$ satisfying the zeroorder compatibility condition, there exists a unique solution $\xi:[0,1] \times[0, \infty)$ to the Cauchy problem (1)-(3).

\section{Moreover, the solution satisfies}

1) if $\xi^{0}(0) \in \mathbb{R}_{+}^{n}$ for all $x \in[0,1]$, then $\xi(x, t) \succeq 0$, for all $(x, t) \in[0,1] \times \mathbb{R}_{+}$;

2) if for all $i=1, \ldots, n \operatorname{row}_{i}(G) \neq 0$, and $\xi^{0}(x) \succ 0$ for all $x \in[0,1]$, then $\xi(x, t) \succ 0$, for all $(x, t) \in$ $[0,1] \times \mathbb{R}_{+}$.

Roughly speaking, besides the existence and uniqueness result of the Cauchy problem (1)-(3), the previous result states the positivity of hyperbolic systems, and that positivity of solutions is preserved for a special kind of boundary condition matrices.

Proof: The well-posedness follows from a classical application of Lumer-Philipps theorem, as done e.g. in [4, Theorem A.1].

For system (1), we can calculate the propagating period of each state component $\xi_{i}(x, t)$ from the boundary $x=0$ to $x=1$ as

$$
\tau_{i}=\frac{1}{\lambda_{i}}, \quad i=1, \ldots, n .
$$

Denote $\underline{\tau}$ be the minimum time of all $\tau_{i}$, i.e., $\underline{\tau}=\min _{i=1, \ldots, n} \tau_{i}$, and for $p \in \mathbb{N}$, let $\Delta_{p} \subset \mathbb{R}_{+}$be defined by

$$
\Delta_{p}=[p \underline{\tau},(p+1) \underline{\tau}] .
$$

We now proceed by induction over the time interval $\Delta_{p}$ to prove Item 1 of Proposition 1 . The first step consists to prove $\xi(x, t) \succeq 0$, for all $t \in \Delta_{0}, x \in[0,1]$. 
For an initial state $\xi^{0} \in \mathbb{R}_{+}^{n}$ in $H^{1}(0,1)$, by the method of characteristics, the state component to the Cauchy problem (1)-(3) on $[0,1] \times \Delta_{0}$ is given as, for all $i=1, \ldots, n$

$$
\begin{cases}\xi_{i}(x, t)= & \\ \sum_{j=1}^{n} g_{i j} \xi_{j}^{0}\left[1-\lambda_{j}\left(t-\frac{x}{\lambda_{i}}\right)\right], & \text { for } \lambda_{i} t>x, \\ \xi_{i}^{0}\left(x-\lambda_{i} t\right), & \text { for } \lambda_{i} t \leq x,\end{cases}
$$

where $g_{i j}$ is the entry $(i, j)$ of the boundary condition matrix $G$.

Since $\xi^{0}(x) \in \mathbb{R}_{+}^{n}$, for all $x \in[0,1]$, and $g_{i j} \geq 0$, it follows from (6) that $\xi(x, t) \succeq 0$, for all $t \in \Delta_{0}$. This concludes the initial step of the induction.

Suppose for $p \geq 0, \xi(x, t) \succeq 0$, for all $t \in \Delta_{p}$. Taking $\xi(\cdot, p T)$ as the initial condition of the system and applying the same argument as above, we get that the solution is uniquely defined, and $\xi(x, t) \succeq 0$ for all $t \in \Delta_{p+1}$.

Therefore, the solution satisfies $\xi(x, t) \succeq 0, t \in \mathbb{R}_{+}$. It concludes the proof of Item 1 in Proposition 1 .

Let us prove Item 2, using again an induction argument. First from (6), noting that all the terms in the sum of the first line of (6) are non-negative and there exist at least one positive term $g_{i j}$ of the sum in (6), we have $\xi_{i}(x, t)>0$, for all $(x, t) \in[0,1] \times \Delta_{0}$. The induction is shown in a similar way taking $\xi(\cdot, p T)$ as an initial condition.

This concludes the proof of Proposition 1.

Remark 1: From Proposition 1, we deduce the fact that the linear hyperbolic system (1)-(3) is positive or not depends only on whether boundary condition matrix $G$ is non-negative.

As the boundary condition matrix $G$ including zero rows, i.e., $\operatorname{row}_{i}(G)=0$, for some row indices $i \in\{1, \ldots, n\}$, the corresponding state $\xi_{i}(x, t)$ becomes zeros in a finite time. Then the rest state components might subsequently becoming zeros under the structure of matrix $G$. In the following, we give an estimation of each state component for system (1)(3), whether it holds positive all the time or when it becomes zero.

Before stating the general results on the solutions of positive linear hyperbolic systems (1)-(3), let us construct a sequence of the index set according to the structure of matrix $G$.

Let $\Omega_{0}=\{1, \ldots, n\}$, and $\mathcal{I}_{1}=\left\{i: \sum_{j \in \Omega_{0}} g_{i j}=0\right\}$ which includes all indices of the zero rows of matrix $G$, i.e., $\operatorname{row}_{i}(G)=0, i=1, \ldots, n$.

We denote the following index sets iteratively, for all $k \in \mathbb{N}$, $k \geq 2$,

$$
\Omega_{k-1}=\{1, \ldots, n\} \backslash \bigcup_{l=1}^{k-1} \mathcal{I}_{l}
$$

and

$$
\mathcal{I}_{k}=\left\{i: i \in \Omega_{k-1}, \text { and } \sum_{j \in \Omega_{k-1}} g_{i j}=0\right\} .
$$

Then, $\mathcal{I}_{k}$ is the largest subset in $\Omega_{k-1}$ such that $\sum_{j \in \Omega_{k-1}} g_{i j}=0$ for all $i \in \mathcal{I}_{k}$, and for all indices $i \notin \mathcal{I}_{k}$ it holds $\sum_{j \in \Omega_{k-1}} g_{i j} \neq 0$.

Since $G$ is a $n$-dimension matrix, the construction process (7)-(8) could be finished in a finite number of iterative steps, that is the index sequence $\mathcal{I}_{k}, k=1, \ldots$, is a finite set, and includes at most $n$ subsets. Let $K$ be such that the last subset $\mathcal{I}_{K}$ is empty, $\mathcal{I}_{K}=\emptyset$, and other sets $\mathcal{I}_{1}, \cdots, \mathcal{I}_{K-1}$ are nonempty.

Item 2 in Proposition 1 indicates that the positive linear hyperbolic systems (1)-(3) might experience a positive solution as the boundary condition matrix $G$ does not include nonzero rows. Given a submatrix $G_{\Omega_{k}}$ (the subscripts instruct the rows and columns of $G$ that make up the square submatrix), $k=0,1, \ldots, K-1$, we prove that the state components $\xi_{i}$, whose index $i$ belongs to the subset $\Omega_{k}$, remains positive as the submatrix $G_{\Omega_{k}}$ does not have non-zero rows. To be more specific the following proposition holds.

Proposition 2: Assume that the matrix $G$ includes zero rows, i.e., row $_{i}(G)=0$, for some $i \in\{1, \ldots, n\}$, and the sequence of index sets $\mathcal{I}_{k}, k=1, \ldots, K$, is defined as (8), $\mathcal{I}_{K}=\emptyset$. Then, for any initial condition $\xi^{0}$ in $H^{1}(0,1)$ satisfying the zero-order compatibility condition and $\xi^{0}(x) \succ 0$, $x \in[0,1]$, the solution to the system (1)-(3) satisfies

1) $\forall i \in \mathcal{I}_{K_{0}}, K_{0}=1, \ldots, K-1$,

$$
\xi_{i}(x, t)=0
$$

for all $(x, t) \in[0,1] \times\left(\sum_{k=1}^{K_{0}} \max _{i \in \mathcal{I}_{k}}\left(\tau_{i}\right),+\infty\right)$, where $\tau_{i}$ is defined by (4);

2) $\forall j \in \Omega_{K-1}$,

$$
\xi_{j}(x, t)>0
$$

for all $(x, t) \in[0,1] \times \mathbb{R}_{+}$.

Proof: 1) Let us prove Item 1 successively for $K_{0}=$ $1,2, \ldots, K-1$.

Since matrix $G$ includes some zero rows, that is $\mathcal{I}_{1} \neq \emptyset$, then for all indices $i \in \mathcal{I}_{1}$, and $t \in \mathbb{R}_{+}$, at the left boundary we have $\xi_{i}(0, t)=0$. Hence, for all $i \in \mathcal{I}_{1}$ and $x \in[0,1]$,

$$
\xi_{i}(x, t)=0, \quad t \in\left(\max _{i \in \mathcal{I}_{1}}\left(\tau_{i}\right),+\infty\right) .
$$

This is (9) for $K_{0}=1$.

For the index $i \in \mathcal{I}_{2}$, it holds $\sum_{j \in \Omega_{1}} g_{i j}=0$. Since $g_{i j} \geq 0$, it follows that $g_{i j}=0$, for all $i \in \mathcal{I}_{2}$, and $j \in \Omega_{1}$. Then, due to $(2)$,

$$
\begin{aligned}
\xi_{i}(0, t) & =\sum_{j \in \mathcal{I}_{1}} g_{i j} \xi_{j}(1, t)+\sum_{j \in \Omega_{1}} g_{i j} \xi_{j}(1, t) \\
& =\sum_{j \in \mathcal{I}_{1}} g_{i j} \xi_{j}(1, t) .
\end{aligned}
$$

From (11), for the indices $j \in \mathcal{I}_{1}$, at the right boundary, we have $\xi_{j}(1, t)=0$ when $t>\max _{i \in \mathcal{I}_{1}}\left(\tau_{i}\right)$. Substituting into (12), it yields $\xi_{i}(0, t)=0$, for all $t \in\left(\max _{i \in \mathcal{I}_{1}}\left(\tau_{i}\right),+\infty\right)$.

Thus, for all $i \in \mathcal{I}_{2}$ and $x \in[0,1]$,

$$
\xi_{i}(x, t)=0, \quad t \in\left(\sum_{k=1}^{2} \max _{i \in \mathcal{I}_{k}}\left(\tau_{i}\right),+\infty\right) .
$$

This is (9) for $K_{0}=2$.

Repeating above procedures, we prove by the induction that, for each index set $\mathcal{I}_{K_{0}}, K_{0}=1, \ldots, K-1$, the corresponding state components $\xi_{i}(x, t), x \in[0,1]$, with index $i \in \mathcal{I}_{K_{0}}$, become zero in a finite time. It concludes the proof of Item 1 in Proposition 2. 
2) We now proceed by induction over the index set $\Omega_{k}$, $k=1, \ldots, K-1$ to prove that for all $j \in \Omega_{k}, x \in[0,1]$ and $t \in\left[0, \sum_{k=0}^{k} \min _{j \in \Omega_{k}}\left(\tau_{j}\right)\right]$, it holds $\xi_{j}(x, t)>0$.

For the index $i \in \Omega_{1}$, and under the boundary condition (2), we have

$$
\xi_{i}(0, t)=\sum_{j \in \Omega_{0}} g_{i j} \xi_{j}(1, t)
$$

On the other hand, given a positive initial condition $\xi^{0}(x)>$ $0, x \in[0,1]$, it holds $\xi_{j}(1, t)>0$, for all $j \in \Omega_{0}$ and $t \in\left[0, \min _{j \in \Omega_{0}}\left(\tau_{j}\right)\right]$. Substituting into (14), and because $\sum_{j \in \Omega_{1}} g_{i j} \neq 0$, for all $i \in \Omega_{1}$, one gets

$$
\xi_{i}(0, t)>0, \quad t \in\left[0, \min _{j \in \Omega_{0}}\left(\tau_{j}\right)\right] .
$$

Thus, for $i \in \Omega_{1}, x \in[0,1]$, the state component $\xi_{i}(x, t)$ satisfies

$$
\xi_{i}(x, t)>0, \quad t \in\left[0, \min _{j \in \Omega_{0}}\left(\tau_{j}\right)+\min _{j \in \Omega_{1}}\left(\tau_{j}\right)\right] .
$$

This is the first step of the induction.

At the second step, for all $i \in \Omega_{2}$, combining

$$
\xi_{i}(0, t)=\sum_{j \in \mathcal{I}_{1}} g_{i j} \xi_{j}(1, t)+\sum_{j \in \Omega_{1}} g_{i j} \xi_{j}(1, t),
$$

$\sum_{j \in \Omega_{1}} g_{i j} \neq 0$ and (16) for $x=1$, we get, for all $j$ in $\Omega_{1}$,

$$
\xi_{j}(1, t)>0, \quad t \in\left[0, \min _{j \in \Omega_{0}}\left(\tau_{j}\right)+\min _{j \in \Omega_{1}}\left(\tau_{j}\right)\right] .
$$
(17)

Because again $\sum_{j \in \Omega_{1}} g_{i j} \neq 0$, for all $i \in \Omega_{2}$, one gets with

$$
\xi_{i}(0, t)>0, \quad t \in\left[0, \sum_{k=0}^{1} \min _{j \in \Omega_{k}}\left(\tau_{j}\right)\right] .
$$

Hence, with (2), for all $i \in \Omega_{2}, x \in[0,1]$,

$$
\xi_{i}(x, t)>0, \quad t \in\left[0, \sum_{k=0}^{2} \min _{j \in \Omega_{k}}\left(\tau_{j}\right)\right] .
$$

This is the second step of the induction from the first step (the other steps are proven similarly). Therefore, by induction, we prove that, for the index set $\Omega_{K-1}$, for all $i \in \Omega_{K-1}$, $x \in[0,1]$, the state components satisfy

$$
\xi_{i}(x, t)>0, \quad t \in\left[0, \sum_{k=0}^{K-1} \min _{j \in \Omega_{k}}\left(\tau_{j}\right)\right] .
$$

Moreover, due to (2), we have

$$
\begin{aligned}
\xi_{i}(0, t)= & {\left[\sum_{j \in \mathcal{I}_{1}}+\cdots+\sum_{j \in \mathcal{I}_{K-1}}\right] g_{i j} \xi_{j}(1, t) } \\
& +\sum_{j \in \Omega_{K-1}} g_{i j} \xi_{j}(1, t) .
\end{aligned}
$$

Since $\mathcal{I}_{K}=\emptyset$, then, for all $i \in \Omega_{K-1}, \sum_{j \in \Omega_{K-1}} g_{i j} \neq 0$. Taking

$$
\tau^{\prime}=\sum_{k=1}^{K-1} \min _{j \in \Omega_{k}}\left(\tau_{j}\right)
$$

as the initial time, and using (21), we see the solution $\xi$ as a strictly positive initial condition $\xi_{i}\left(x, \tau^{\prime}\right)>0$, for all $x \in$ $[0,1]$.

Following the proof of Item 2 of Proposition 1, for all $i \in$ $\Omega_{K-1}$, we have $\xi_{i}(x, t)>0$, for all $t \geq \tau^{\prime}, x \in[0,1]$.
This concludes the proof of Proposition 2.

Example 1: Let us consider the following non-negative boundary condition matrix

$$
G=\left(\begin{array}{cccc}
0 & 0 & 0 & 0 \\
0 & 0 & 0 & 1 \\
0 & 0 & 1 & 0 \\
1 & 0 & 0 & 0
\end{array}\right) .
$$

We have $\mathcal{I}_{1}=\{1\}, \mathcal{I}_{2}=\{4\}, \mathcal{I}_{3}=\{2\}$, and $\mathcal{I}_{4}=\emptyset$. Thus $\{1,2,3,4\}-\bigcup_{k=1}^{3} \mathcal{I}_{k}=\{3\}$. For any initial condition $\xi^{0}(x) \succ 0$, the third state component satisfies $\xi_{3}(x, t) \succ 0$ for all $(x, t) \in[0,1] \times \mathbb{R}_{+}$. For any $x \in[0,1]$, other state components become zeros in finite time. More precisely, $\xi_{1}(x, t)=0$ when $t \in\left[\tau_{1}, \infty\right), \xi_{4}(x, t)=0$ when $t \in\left[\tau_{1}+\tau_{4}, \infty\right)$, and $\xi_{2}(x, t)=0$ when $t \in\left[\tau_{1}+\tau_{4}+\tau_{2}, \infty\right)$.

Remark 2: We can consider more general positive linear hyperbolic systems with both negative and positive velocities for $\Lambda$, such as with $\lambda_{i}<0$ for $i \in\{1, \ldots, m\}$, and $\lambda_{i}>0$ for $i \in\{m+1, \ldots, n\}$. By defining the state description $\xi=$ $\left(\xi^{-}, \xi^{+}\right)^{\top}$, where $\xi^{-} \in \mathbb{R}^{m}$ and $\xi^{+} \in \mathbb{R}^{n-m}$, and the change of variable $w(x, t)=\left[\xi^{-}(1-x, t), \xi^{+}(x, t)\right]^{\top}$, we can obtain a new hyperbolic system in the same form as (1)-(2).

\section{NeCESSARY AND SUFFICIENT CONDITIONS ON THE EXPONENTIAL STABILITY}

Let us start this section by defining the notion of the exponential stability under consideration in this paper.

Definition 1: The linear hyperbolic system (1)-(2) is said to be exponentially stable in $L^{1}$-norm, if there exist $\nu>0$ and $C>0$ such that, for every initial condition $\xi(\cdot, 0)$ in $H^{1}(0,1)$ satisfying the zero-order compatibility condition, the solution $\xi$ to the Cauchy problem (1)-(3) satisfies, for all $t \in \mathbb{R}_{+}$,

$$
\|\xi(., t)\|_{L^{1}(0,1)} \leq C e^{-\nu t}\|\xi(., 0)\|_{L^{1}(0,1)} .
$$

\section{A. Conditions for the existence of a linear Lyapunov function}

Let us first deal with a sufficient condition for the existence of a linear Lyapunov function yielding an exponential stability of positive linear hyperbolic systems. This sufficient condition is written in terms of a dissipative boundary condition and is also necessary under an additional assumption, as written in the following first main result.

Theorem 1: Consider the positive linear hyperbolic system (1)-(3) with matrix $G \in \mathbb{R}_{+}^{n \times n}$. Let $\theta \in \mathbb{R}_{+}^{n}$ be a positive vector, i.e. $\theta \succ 0$, and $\mu>0$ be a constant such that

$$
\left[G^{\top}-e^{-\mu} I_{n}\right] \theta \preceq 0 .
$$

- The system (1)-(3) is exponentially stable if condition (25) holds, and moreover a Lyapunov function is given by, for all $\xi$ in $L^{1}(0,1)$,

$$
V(\xi)=\int_{0}^{1} \theta^{\top} \Lambda^{-1} \xi e^{-\mu x} d x .
$$

- Conversely, if the system (1)-(3) is exponentially stable and the inverse of velocities, $\lambda_{i}^{-1}$, are commensurable, then there exist $\theta \succ 0$ and $\mu>0$ such that (25) holds. 
Remark 3: Before proving this theorem, let us note that the Lyapunov function candidate $V$ defined in (26) is inspired by [6] among other references where the same weight $x \mapsto$ $\exp (-\mu x)$ is used combined with a $L^{2}$ integral norm (see also [9]). It is also inspired by [19] where the Lyapunov function is linear which implies a prior requirement that all state variables are non-negative. This linear Lyapunov function candidate is not appropriate for general linear hyperbolic systems.

Proof: Sufficiency. Using an integration by part, the time derivative of the Lyapunov function (26) along the solutions to (1) is computed as $\dot{V}=-\int_{0}^{1} \theta^{\top} \partial_{x} \xi e^{-\mu x} d x=$ $-\left[\theta^{\top} \xi e^{-\mu x}\right]_{0}^{1}-\mu \int_{0}^{1} \theta^{\top} \xi e^{-\mu x} d x$. Now under the boundary condition (2), it holds $\dot{V}=\xi^{\top}(1, t)\left[G^{\top}-e^{-\mu} I_{n}\right] \theta-$ $\mu \int_{0}^{1} \theta^{\top} \xi e^{-\mu x} d x$.

Moreover, using $G$ in $\mathbb{R}_{+}^{n}$ and Proposition 1, we have $\xi(1, t) \in \mathbb{R}_{+}^{n}$ for all $t \geq 0$ and thus $\dot{V} \leq-\mu \underline{\lambda} V(\xi)$ where $\underline{\lambda}=\min \left\{\lambda_{i}, i=1, \ldots, n\right\}$. By remarking that there exist positive values $C_{1}$ and $C_{2}$ (depending on $\theta, \Lambda$, and $\mu$ ) such that, for all non-negative functions $\xi$, it holds $C_{1}\|\xi\|_{L^{1}(0,1)} \leq$ $V(\xi) \leq C_{2}\|\xi\|_{L^{1}(0,1)}$, we may deduce that the solutions of the positive linear hyperbolic system (1) exponentially converge to 0 in $L^{1}$-norm.

This complete the proof of sufficiency.

Necessity. Since the inverse of the velocities, $\lambda_{i}^{-1}$, are commensurable, there exists a sufficiently small time-interval $\Delta t \in \mathbb{R}_{+}$, such that the time periods satisfy $\tau_{j}=d_{j} \Delta t$, for suitable integers $d_{j} \in \mathbb{N}, j=1, \ldots, n$.

Then, we could find a common time sample at which the dynamics of each state component $\xi_{i}(x, t)$ project to its left boundary. Roughly speaking the necessary condition is later derived by considering the time evolution of $\xi_{i}(0, t)$, for all $t \geq 0$.

Let $\bar{\tau}$ be the maximum time of all $\tau_{i}$, i.e., $\bar{\tau}=\max _{i=1, \ldots, n} \tau_{i}$. Using that state $\xi_{i}$ is constant along the characteristic curves, the values of every state component at the right boundary at time $t$, are equivalent to those at time $t-\tau_{i}$, that is,

$$
\xi_{i}(1, t)=\xi_{i}\left(0, t-\tau_{i}\right),
$$

for all $t \geq \tau_{i}, i=1, \ldots, n$.

Combined with the boundary condition (2), for $t \geq \bar{\tau}$, the dynamics of system (1) at the left boundary $x=0$ can be represented as

$$
\xi(0, t)=\sum_{j=1}^{n} G[j] \xi\left(0, t-\tau_{j}\right),
$$

where $G[j]=\left[0, \ldots, \operatorname{col}_{j}(G), \ldots, 0\right]$ is a $n$-dimension square matrix, and $\operatorname{col}_{j}(G)$ is the $j$-th column of the matrix $G$. We find $\sum_{j=1}^{n} G[j]=G$. Using the notation $l=\frac{\bar{\tau}}{\Delta t}$, we note that $d_{j} \leq l$ for all $j=1, \ldots, n$, and thus we get the following time-delay equation from (28),

$$
\xi(0, s \Delta t)=\sum_{j=1}^{n} G[j] \xi\left(0, s \Delta t-d_{j} \Delta t\right),
$$

for $s=l, l+1, \ldots$.

Simply denote $y_{s}=\xi(0, s \Delta t)$. By reorganizing the terms in (29), we get the following time-delay system, for $s=l, l+$
$1, \ldots$,

$$
y_{s}=\sum_{d=1}^{l} \mathcal{G}_{d} y_{s-d}
$$

where

$$
\mathcal{G}_{d}=\left\{\begin{array}{cl}
\sum_{d_{j}=d} G[j] & \text { there exists } j \text { such that } d=d_{j} \\
0 & \text { otherwise }
\end{array}\right.
$$

and initial values $y_{l-d}=\xi(0,(l-d) \Delta t)$, for all $d=1, \ldots, l$, is uniquely defined with Proposition 1. Further, with Proposition 2 , the state components $\xi_{i}(0, s \Delta t)>0$, as $i \in \Omega_{K-1}$, and the others keep non-negative.

Now, we consider the discrete-time instant

$$
h=\frac{1}{\Delta t} \sum_{i=1}^{n} \tau_{i} .
$$

From (30), for all $s=h+2, \ldots, h+p,(p \in \mathbb{N})$, we have, respectively $y_{h+2}=\sum_{d=1}^{l} \mathcal{G}_{d} y_{h+2-d}, \ldots, y_{h+p}=$ $\sum_{d=1}^{l} \mathcal{G}_{d} y_{h+p-d}$. Summing the above equalities one obtains

$$
\begin{aligned}
\sum_{s=h+2}^{h+p} y_{s} & =\sum_{s=h+2}^{h+p} \sum_{d=1}^{l} \mathcal{G}_{d} y_{s-d}=\sum_{d=1}^{l} \mathcal{G}_{d} \sum_{s=h+2}^{h+p} y_{s-d} \\
& =\sum_{d=1}^{l} \mathcal{G}_{d}\left[\sum_{s=h+2}^{h+p-d} y_{s}+\sum_{s=h+2-d}^{h+1} y_{s}\right] .
\end{aligned}
$$

Since the system (1)-(3) is exponentially stable in $L^{1}$-norm, then for all $x \in(0,1)$, except on a set of measure zero, $\xi(x, t) \rightarrow 0$, as $t \rightarrow \infty$, exponentially fast. Since the solution is continuous with respect to $x$, this implies the exponential pointwise convergence of $\xi(x, t) \rightarrow 0$, as $t \rightarrow \infty$, for all $x$ in $(0,1)$. In particular, $\xi(0, t) \rightarrow 0$, exponentially fast, as $t \rightarrow \infty$. For the corresponding discrete system (30), $\sum_{s=h+2}^{\infty} y_{k}<\infty$. It follows from (32)

$$
\begin{aligned}
-\sum_{d=1}^{l} \mathcal{G}_{d} \sum_{s=h+2-d}^{h+1} y_{s} & =\left[\sum_{d=1}^{l} \mathcal{G}_{d}-I_{n}\right] \sum_{s=h+2}^{\infty} y_{s} \\
& =\left[G-I_{n}\right] \sum_{s=h+2}^{\infty} y_{s} .
\end{aligned}
$$

Moreover, due to Item 1 of Proposition 1, all the terms in the left-hand side of equality (33) are non-positive, we have

$$
\begin{aligned}
{\left[G-I_{n}\right] \sum_{s=h+2}^{\infty} y_{s} } & \preceq-\sum_{d=1}^{l} \mathcal{G}_{d} y_{h+1} \\
& \preceq-G y_{h+1} .
\end{aligned}
$$

To conclude the necessity part of the proof, and show that inequality (25) holds for suitable $\theta \succ 0$ and $\mu>0$, two cases about matrix $G$ may occur:

Case 1: $\operatorname{row}_{i}(G) \neq 0$, for all $i=1, \ldots, n$.

According to Item 2 in Proposition 1, the solution of the Cauchy problem (1)-(3) with an positive initial condition $\xi^{0}(x) \succ 0$ satisfies $\xi(x, t) \succ 0$ for all $(x, t) \in[0,1] \times \mathbb{R}_{+}$. 
In particular $y_{s} \succ 0$, for all $s \geq h+1$. Thus $-G y_{h+1} \prec 0$. With (34), it holds

$$
\left[G-I_{n}\right] b \prec 0,
$$

by letting $b=\sum_{s=h+2}^{\infty} y_{s} \succ 0$.

Moreover, there exists $\mu>0$ such that

$$
\left[G-e^{-\mu} I_{n}\right] b \preceq 0 .
$$

Because $-\left[G-e^{-\mu} I_{n}\right]$ is a M-matrix and $b \succ 0$ satisfying (36), there exists a positive vector $\theta \in \mathbb{R}_{+}^{n}, \theta \succ 0$, such that $\left[G^{\top}-e^{-\mu} I_{n}\right] \theta \preceq 0$.

Case 2: There exists at least one row of indices $i \in$ $\{1, \ldots, n\}$, such that $\operatorname{row}_{i}(G)=0$.

Assume the sequence of index set $\mathcal{I}_{k}$ as (8) is constructed, $k=1, \ldots, K-1$, and with an initial condition to (1)-(3) starting from $\xi^{0}(x) \succ 0$ for all $x \in[0,1]$.

Since $h=\frac{1}{\Delta t} \sum_{i=1}^{n} \tau_{i}$, we have

$$
h \geq \frac{1}{\Delta t} \sum_{k=1}^{K-1} \max _{i \in \mathcal{I}_{k}}\left(\tau_{i}\right) .
$$

Therefore according to Proposition 2, for all $s \geq h+1$, it follows, $\left(y_{s}\right)_{\mathcal{I}_{k}}=0$, for all $k=1, \ldots, K-1$, and $\left(y_{s}\right)_{\Omega_{K-1}} \succ$ 0 .

Because again $b=\sum_{s=h+2}^{\infty} y_{s}$ is constant, it follows $b_{\mathcal{I}_{k}}=$ 0 , for all $k=1, \ldots, K-1$, and $b_{\Omega_{K-1}} \succ 0$.

Furthermore, under the structure of matrix $G$, the $(i, i)$ entries of $\left[G-I_{n}\right]_{\mathcal{I}_{k}}$ (a submatrix of $\left[G-I_{n}\right]$ whose row indices belong to $\mathcal{I}_{k}$ ) is $-1, i \in \mathcal{I}_{k}$, and the $(i, j)$ entries of $\left[G-I_{n}\right]_{\mathcal{I}_{k}}$ is 0 , for $i \in \mathcal{I}_{k}, j \in \Omega_{k}, k=1, \ldots, K-1$. Hence, we have, for all $k=1, \ldots, K-1$,

$$
\left(\left[G-I_{n}\right] b\right)_{\mathcal{I}_{k}}=0 .
$$

On the other hand, from inequality (34), we have

$$
\left(\left[G-I_{n}\right] b\right)_{\Omega_{K-1}} \preceq\left(-G y_{h+1}\right)_{\Omega_{K-1}} \prec 0 .
$$

Let vector $\tilde{b}$ in $\mathbb{R}^{n}$ such that $\tilde{b}_{\mathcal{I}_{k}}=\epsilon_{k}$, for all $k=$ $1, \ldots, K-1$, and $\tilde{b}_{\Omega_{K-1}}=0$ for the remaining entries of $\tilde{b}, \epsilon_{k}$ are sufficiently small positive values satisfying

$$
\epsilon_{k}>\max _{i \in \mathcal{I}_{k}}\left\{\sum_{r=1}^{k} \sum_{j \in \mathcal{I}_{r}} g_{i j} \epsilon_{r}\right\} .
$$

Hence, $b+\tilde{b} \succ 0$, and, due to (38) and (39), $\left[G-I_{n}\right](b+$ $\tilde{b}) \prec 0$ holds. The remaining proof of this case is as in the first case.

It concludes the proof of Theorem 1.

\section{B. Conditions for the existence of a quadratic Lyapunov function}

Before commenting on the second main result, let us recall the following sufficient condition for the existence of a quadratic Lyapunov function.

Proposition 3: [8] Let $P \in \mathbb{R}^{n \times n}$ be a diagonal positive definite matrix, $\mu>0$ be a constant, the function

$$
V(\xi)=\int_{0}^{1} \xi^{\top} \Lambda^{-1} P \xi e^{-2 \mu x} d x,
$$

is a quadratic Lyapunov function for system (1)-(3), that is $\dot{V} \leq-\frac{2 \mu}{\left(\min _{i} \lambda_{i}\right)^{-1}} V$ along the solutions to (1)-(3), if the matrix inequality

$$
e^{-2 \mu} P-G^{\top} P G \geq 0
$$

holds.

The second main result, namely Theorem 2 below, proves that the sufficient condition for the existence of a linear Lyapunov function as written in item 1) of Theorem 1 is equivalent to the dissipative boundary condition (42) considered in Proposition 3. In details, Theorem 2 below gives three characterizations for the matrix inequality (42) to hold, each of them implying the existence of a quadratic Lyapunov function for system (1)-(3).

Theorem 2: Given a positive linear hyperbolic system (1)(3), the following are equivalent:

1) there exist $\mu>0$, and a vector $\theta \succ 0$, such that condition (25) holds;

2) there exist $\mu>0$ and a diagonal positive definite matrix $P>0$, such that inequality (42) holds;

3) $\rho(G)<1$, where $\rho(G)$ is the spectral radius of the matrix $G$.

Proof: 1) $\Rightarrow$ 2) We denote $\left[G^{\top}-e^{-\mu} I_{n}\right] \theta=v \preceq 0$, and $\left[G-e^{-\mu} I_{n}\right] b=u \preceq 0$, where $\theta=\left[\theta_{1}, \ldots, \theta_{n}\right]^{\top}$, and $b=\left[b_{1}, \ldots, b_{n}\right]^{\top}$.

Using $\theta$ and $b$, we construct a diagonal positive definite matrix as $P=\operatorname{diag}\left\{\frac{\theta_{1}}{b_{1}}, \frac{\theta_{2}}{b_{2}}, \ldots, \frac{\theta_{n}}{b_{n}}\right\}$. We have $P b=\theta$ and $G b=u+e^{-\mu} b$. Then

$$
\begin{aligned}
\left(e^{-2 \mu} P-G^{\top} P G\right) b & =e^{-2 \mu} \theta-G^{\top} P\left(u+e^{-\mu} b\right) \\
& =e^{-\mu}\left(e^{-\mu} I_{n} \theta-G^{\top} \theta\right)-G^{\top} P u \\
& =-e^{-\mu} v-G^{\top} P u \succeq 0 .
\end{aligned}
$$

Since $e^{-2 \mu} P-G^{\top} P G$ is a symmetric M-matrix and satisfies inequality (43), it must be a semi-definite positive matrix, and thus (42) holds.

$2) \Rightarrow 3$ ) Using the congruence transformation defined by the positive definite matrix $e^{-\mu} P^{\frac{1}{2}}$ on both sides of $e^{-2 \mu} P-$ $G^{\top} P G=Q$, yields

$$
\begin{array}{r}
\left(e^{\mu} P^{-\frac{1}{2}} G^{\top} P^{\frac{1}{2}}\right)\left(P^{\frac{1}{2}} G P^{-\frac{1}{2}} e^{\mu}\right)-I_{n} \\
=-\left(e^{\mu} P^{-\frac{1}{2}}\right) Q\left(P^{-\frac{1}{2}} e^{\mu}\right) .
\end{array}
$$

Since the right-hand side of (44) is semi-definite negative, this requires that $\left\|P^{\frac{1}{2}} G P^{-\frac{1}{2}} e^{\mu}\right\|_{2} \leq 1$. Thus, with $\mu>0$, $\rho(G)=\rho\left(P^{\frac{1}{2}} G P^{-\frac{1}{2}}\right) \leq\left\|P^{\frac{1}{2}} G P^{-\frac{1}{2}}\right\|_{2}<1$.

$3) \Rightarrow 1$ ) follows from the Perron-Frobenius theorem.

This concludes the proof of Theorem 2 .

Example 2: Consider a positive linear hyperbolic system (1)-(2) with the characteristic and the boundary condition matrices given, respectively, by $\Lambda=\operatorname{diag}\{1,-1,2\}$, and

$$
G=\left[\begin{array}{ccc}
0.2 & 0.4 & 0.2 \\
0.8 & 0.2 & 0.1 \\
0.4 & 0 & 0.2
\end{array}\right] .
$$

The spectral radius of the non-negative matrix $G$ is $\rho(G)=$ $0.8990<1$. After solving the linear inequality condition $(25)$, one can easily construct a linear Lyapunov function (26) with 
$\theta=(6,4,3)^{\top} \succ 0$ and $\mu=0.01>0$. Following the results of Theorem 2, a quadratic Lyapunov function (41) can be further constructed with $b=(4,5,5)^{\top} \succ 0$ and $P=\operatorname{diag}\{1.5,0.8,0.6\}>0$.

\section{Application to Freeway TrafFic Control}

To illustrate the usefulness of the results, we will consider the stabilization problem of vehicle transportation systems in a freeway link which is modeled by the positive hyperbolic linear systems.

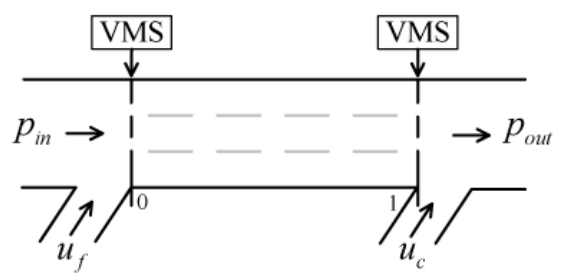

Fig. 1. A freeway link controlled by local on-ramp metering and variable speed sign.

A homogeneous freeway section between two successive on-ramps is sketched in Fig. 1, where $u_{f}$ and $u_{c}$ are two boundary feedback laws that will be defined in this section. We assume that the upstream and the downstream boundaries of the freeway section are provided with the on-ramp metering to regulate the flow-rate of driving-in vehicles, and with the VMSs to limit the driving speed of the mainline traffic.

The control of the freeway traffic is defined from upstream to downstream and regulates the traffic limit of the freeway section (see Section IV-C below for more details). Usually the transmission speed of information from upstream to downstream is faster than the speed of the vehicles in the freeway. Therefore, for this application, the transmission delay will be neglected.

\section{A. Aw-Rascle traffic flow model}

The traffic dynamics of the freeway link are described by a system of two laws of conservation, the so-called Aw-Rascle traffic flow model [2]. It is

$$
\left\{\begin{array}{l}
\partial_{t} \rho+\partial_{x}(\rho v)=0 \\
\partial_{t}(v+p(\rho))+v \partial_{x}(v+p(\rho))=0
\end{array},\right.
$$

where $\rho(x, t)$ is the vehicle density, $v(x, t)$ is the average speed, $x \in[0,1], t \geq 0$, and $p(\rho)$ means the traffic pressure term, which is supposed to increase over the vehicle density.

In [26], a special pressure function $p(\rho)$ is given as

$$
p(\rho)=v_{f}-V(\rho),
$$

where $v_{f}$ is the free (maximal) speed, and $V(\rho)$ is the speeddensity fundamental diagram. Typically, with the Greenshields fundamental diagram [12],

$$
V(\rho)=v_{f}\left(1-\frac{\rho}{\rho_{m}}\right),
$$

where $\rho_{m}$ is the maximal density. Thus, we have $p(\rho)=a \rho$, and $a=\frac{v_{f}}{\rho_{m}}$.
Let $w=v+a \rho, z=v$. The nonlinear hyperbolic equation (46) may be written in the characteristic Riemann coordinates as

$$
\left\{\begin{array}{l}
\partial_{t} w+z \partial_{x} w=0 \\
\partial_{t} z+(2 z-w) \partial_{x} z=0
\end{array} .\right.
$$

In (49), the first velocity is assumed to be positive $z>0$, and the second velocity $2 z-w$ is assumed to be nonzero and its sign does not change. The sign of the second velocity indicates the transfer direction of the vehicle speed from the freeway upstream section to the downstream, or inverse. It is usually used as the feature to determine the freeway traffic lies in the free-flow mode or in the congestion mode [16]. In practice, it also determines how to regulate the freeway traffic by using the upstream or the downstream traffic measurements (see Section V-C below).

\section{B. Steady-state and linearization}

A steady-state of the freeway traffic is a constant traffic state $\left(\rho^{*}, v^{*}\right)$ which satisfies one of the following relation

$$
\begin{aligned}
p_{\text {in }}+r_{f} & =\rho^{*} v^{*}, \quad \text { or } \\
\rho^{*} v^{*} & =p_{\text {out }}-r_{c},
\end{aligned}
$$

where $p_{i n}, r_{f}$ are constant flow-rates of the driving-in vehicles through the upstream mainline and the upstream on-ramp of the freeway section, and $p_{\text {out }}, r_{c}$ are constant driving-out flowrates of the downstream mainline and on-ramp.

In order to linearize the model (46), we define the deviations of the state $\rho(x, t), v(x, t)$ with respect to the steady-state as, respectively

$$
\begin{aligned}
& \hat{\rho}=\rho-\rho^{*}, \\
& \hat{v}=v-v^{*} .
\end{aligned}
$$

The linearization of the system (49) with the steady-state $\left(w^{*}, z^{*}\right)$ is written as

$$
\left\{\begin{array}{l}
\partial_{t} \hat{w}+\left(z^{*}+\hat{z}\right) \partial_{x} \hat{w}=0 \\
\partial_{t} \hat{z}+\left(2 z^{*}-w^{*}+2 \hat{z}-\hat{w}\right) \partial_{x} \hat{z}=0
\end{array} .\right.
$$

In above Riemann coordinates, the deviations are $\hat{w}=w-w^{*}$, and $\hat{z}=z-z^{*}$, with the associated steady-state given as $w^{*}=v^{*}+a \rho^{*}, z^{*}=v^{*}$.

For the traffic steady-state we only assume that the product of vehicle density times speed, that is the flow-rate, is constant. Then there exists a set of the steady-states $\left(\rho^{*}, v^{*}\right)$ satisfying the condition (50). In fact, since

$$
\lambda_{2}^{*}=2 z^{*}-w^{*}=v^{*}-a \rho^{*},
$$

we regard just as two categories of the traffic steady-states. While the steady-state $\left(\rho^{*}, v^{*}\right)$ satisfies $v^{*}-a \rho^{*}>0$, we call it the free-flow steady-state, while $v^{*}-a \rho^{*}<0$, the congestion steady-state.

\section{Boundary feedback control}

Now we are going to show how Theorem 2 may be applied to analyze the local stability of the freeway traffic under the boundary feedback control.As mentioned above, the regulation strategies (using different controller and measurement) are 
designed depending on the steady-state of the freeway traffic lying in the free-flow or congestion traffic modes.

Two cases are discussed separately.

Case 1: $\lambda_{2}^{*}=2 z^{*}-w^{*}>0$, the steady-state $\left(\rho^{*}, v^{*}\right)$ lies in the free-flow mode.

In this case, the velocity information ( $z$ or $v$ ) is propagating from upstream to downstream, and thus it is natural to control $u_{f}$ and $v(0, t)$. We assume that the vehicle density $\rho(1, t)$ and the average speed $v(1, t)$ at the downstream boundary are measured, and the control units are the upstream on-ramp metering and the driving-in speed limit of the freeway section.

Precisely, we introduce the boundary feedback law:

$$
\left\{\begin{array}{l}
u_{f}(t)=r_{f}+k_{\rho}^{f}\left(\rho(1, t)-\rho^{*}\right) \\
v(0, t)=v^{*}+k_{v}^{f}\left(v(1, t)-v^{*}\right)
\end{array},\right.
$$

with feedback gains $k_{\rho}^{f}>0$ and $k_{v}^{f}>0$. The previous controllers are proportional controllers that do not need the knowledge of the entire state, but only the measurement of the vehicle density $\rho(1, t)$ and the average speed $v(1, t)$ at the downstream boundary.

At the upstream boundary of the freeway section, i.e., $x=$ 0 , the driving-in flow-rate conservation equation holds

$$
u_{f}(t)+p_{i n}=\rho(0, t) v(0, t) .
$$

After the linearization of the boundary condition (55) with integrating the feedback control law (54), we have the following boundary condition

$$
\hat{\rho}(0, t)=\frac{k_{\rho}^{f}}{v^{*}} \hat{\rho}(1, t)-\rho^{*} \frac{k_{v}^{f}}{v^{*}} \hat{v}(1, t) .
$$

Since $\hat{v}(1, t)=\hat{z}(1, t)$ and $\hat{\rho}(x, t)=(\hat{w}(x, t)-\hat{v}(x, t)) / a$, as $x=0,1$, we could rewrite condition (56) in the Riemann coordinates for the system (52) as

$$
\begin{aligned}
\hat{w}(0, t)= & \frac{k_{\rho}^{f}}{v^{*}} \hat{w}(1, t) \\
& +\left[k_{v}^{f}-a \rho^{*} \frac{k_{v}^{f}}{v^{*}}-\frac{k_{\rho}^{f}}{v^{*}}\right] \hat{z}(1, t) .
\end{aligned}
$$

Then the boundary condition that need to be imposed for the system (52) is written as

$$
\left[\begin{array}{c}
\hat{w}(0, t) \\
\hat{z}(0, t)
\end{array}\right]=G_{f}\left[\begin{array}{c}
\hat{w}(1, t) \\
\hat{z}(1, t)
\end{array}\right],
$$

where

$$
G_{f}=\left[\begin{array}{cc}
\frac{k_{\rho}^{f}}{v^{*}} & k_{v}^{f}-a \rho^{*} \frac{k_{v}^{f}}{v^{*}}-\frac{k_{\rho}^{f}}{v^{*}} \\
0 & k_{v}^{f}
\end{array}\right] .
$$

In the boundary condition matrix $G_{f}$,

$$
k_{v}^{f}-a \rho^{*} \frac{k_{v}^{f}}{v^{*}}-\frac{k_{\rho}^{f}}{v^{*}}=\frac{\lambda_{2}^{*} k_{v}^{f}-k_{\rho}^{f}}{v^{*}} .
$$

Let us choose $\lambda_{2}^{*} k_{v}^{f}-k_{\rho}^{f} \geq 0$, the boundary condition matrix $G_{f}$ is non-negative, i.e., $G_{f} \succeq 0$. Therefore, applying Proposition 1, system (52) with the boundary condition (58) is a positive linear hyperbolic system.

Straightforward calculations show that Theorem 1 holds for the matrix $G_{f}$, if and only if, $k_{\rho}^{f}, k_{v}^{f}$ of the feedback control law (54) can be selected as $0<k_{\rho}^{f}<v^{*}, 0<k_{v}^{f}<1$, and $\lambda_{2}^{*} k_{v}^{f}-k_{\rho}^{f} \geq 0$.

Case 2: $\lambda_{2}^{*}=2 z^{*}-w^{*}<0$, the steady-state $\left(\rho^{*}, v^{*}\right)$ lies in the congestion mode.

In this case, velocity information is propagating from upstream to downstream and thus it is natural to control $u_{c}$ and $v(1, t)$. Thus, conversely, the measurements are the vehicle density $\rho(0, t)$ and the average speed $v(0, t)$ at the upstream boundary, and the control units are the downstream on-ramp metering and the driving-out speed limit of the freeway section.

Then, we introduce the other boundary feedback law:

$$
\left\{\begin{array}{l}
u_{c}(t)=r_{c}-k_{\rho}^{c}\left(\rho(0, t)-\rho^{*}\right) \\
v(1, t)=v^{*}+k_{v}^{c}\left(v(0, t)-v^{*}\right)
\end{array},\right.
$$

with feedback gains $k_{\rho}^{c}>0$ and $k_{v}^{c}>0$. The previous controllers are again proportional controllers that need only the measurements of the vehicle density $\rho(0, t)$ and the average speed $v(0, t)$ at the upstream boundary.

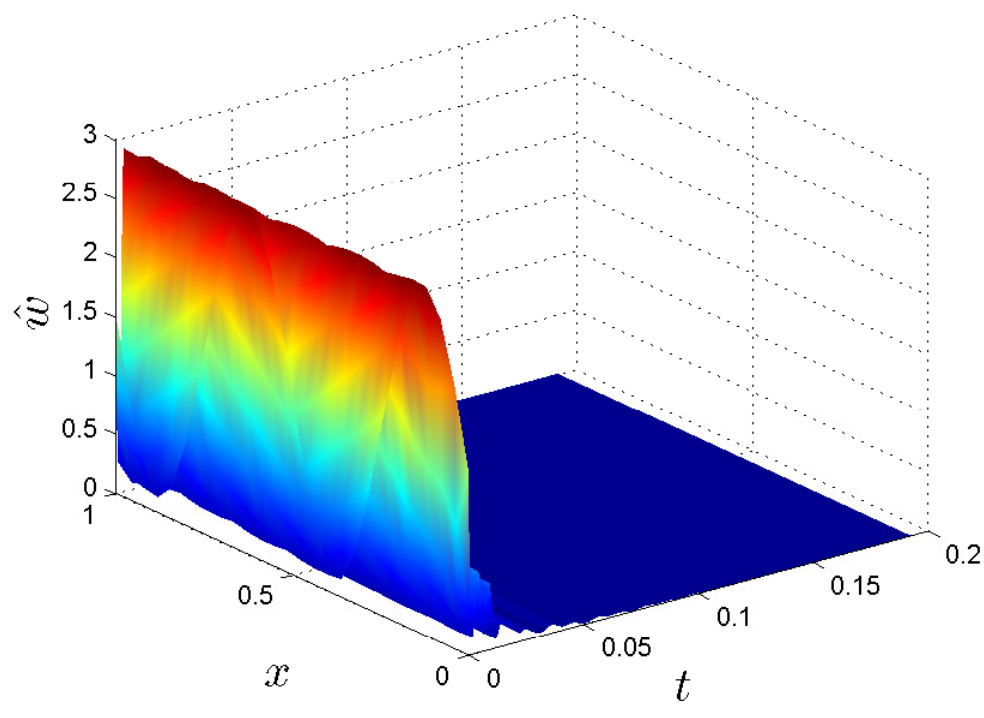

Fig. 2. The evolution of the first component $\hat{w}$ of the positive linear hyperbolic system (52) with matrix $G_{f}$.

At the right boundary of the freeway link, i.e., $x=1$, the driving-out flow-rate conservation equation holds

$$
p_{\text {out }}-u_{c}(t)=\rho(1, t) v(1, t) .
$$

Combining the boundary condition (62) with the feedback control law (61), we have

$$
\hat{\rho}(0, t)=\frac{v^{*}}{k_{\rho}^{c}} \hat{\rho}(1, t)+\rho^{*} \frac{k_{v}^{c}}{k_{\rho}^{c}} \hat{v}(0, t) .
$$

Under the Riemann coordinates, since $\hat{v}(0, t)=\hat{z}(0, t)$ and $\hat{\rho}(x, t)=(\hat{w}(x, t)-\hat{v}(x, t)) / a$, as $x=0,1$, then it holds

$$
\begin{aligned}
\hat{w}(0, t)= & \frac{v^{*}}{k_{\rho}^{c}} \hat{w}(1, t) \\
& +\left[1+a \rho^{*} \frac{k_{v}^{c}}{k_{\rho}^{c}}-v^{*} \frac{k_{v}^{c}}{k_{\rho}^{c}}\right] \hat{z}(0, t) .
\end{aligned}
$$



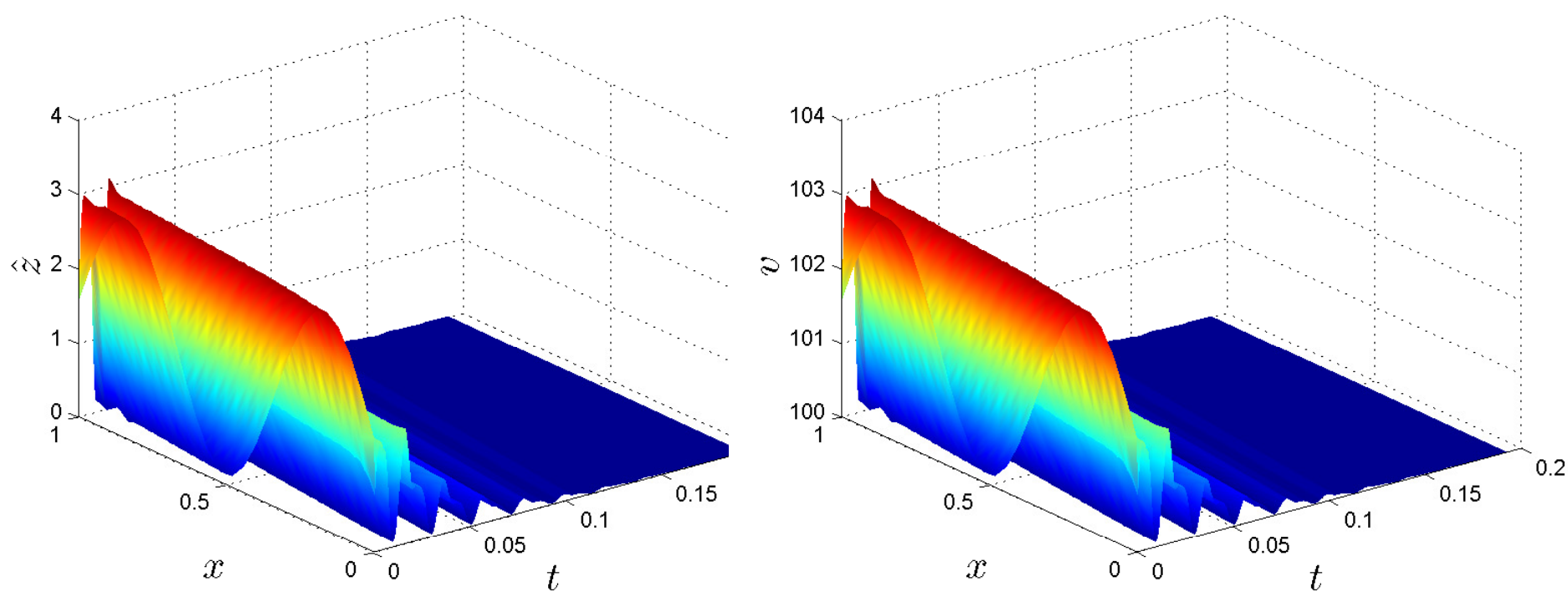

Fig. 3. The evolution of the second component $\hat{z}$ of the positive linear hyperbolic system (52) with matrix $G_{f}$.

Fig. 5. The evolution of average speed $v$ of the Aw-Rascle traffic flow equation (46) with the steady-state $\left(\rho_{1}^{*}, v_{1}^{*}\right)$.

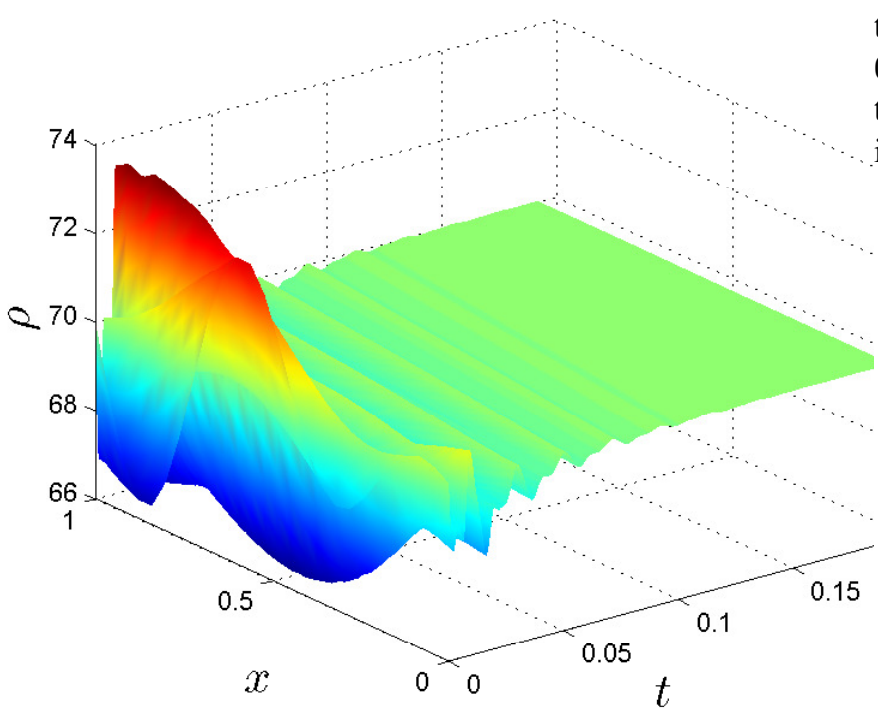

Fig. 4. The evolution of the density $\rho$ of the Aw-Rascle traffic flow equation (46) with the steady-state $\left(\rho_{1}^{*}, v_{1}^{*}\right)$.

The corresponding boundary condition for the system (52) is given as

$$
\left[\begin{array}{c}
\hat{w}(0, t) \\
\hat{z}(1, t)
\end{array}\right]=G_{c}\left[\begin{array}{c}
\hat{w}(1, t) \\
\hat{z}(0, t)
\end{array}\right]
$$

where

$$
G_{c}=\left[\begin{array}{cc}
\frac{v^{*}}{k_{\rho}^{c}} & 1+a \rho^{*} \frac{k_{v}^{c}}{k_{\rho}^{c}}-v^{*} \frac{k_{v}^{c}}{k_{\rho}^{c}} \\
0 & k_{v}^{c}
\end{array}\right] .
$$

Because

$$
1+a \rho^{*} \frac{k_{v}^{c}}{k_{\rho}^{c}}-v^{*} \frac{k_{v}^{c}}{k_{\rho}^{c}}=1-\lambda_{2}^{*} \frac{k_{v}^{c}}{k_{\rho}^{c}}>0,
$$

the boundary condition matrix $G_{c}$ is non-negative, i.e., $G_{c} \succeq$ 0 , and the solution to system (52) is non-negative for all the time, if only $k_{\rho}^{c}>0$ and $k_{v}^{c}>0$, and if the initial condition is non-negative.

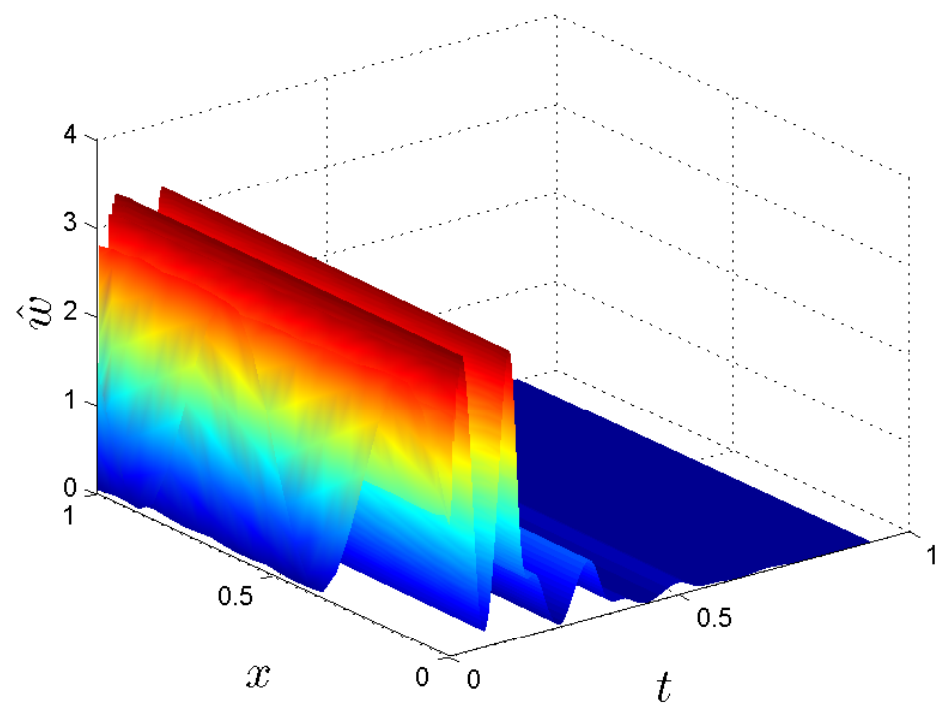

Fig. 6. The evolution of the first component $\hat{w}$ of the positive linear hyperbolic system (52) with matrix $G_{c}$.

Moreover, as the freeway traffic is lying in the congestion mode, the system (52) with the boundary condition matrix $G_{c}$ is locally exponentially stable if and only if the control gains $k_{\rho}^{c}, k_{v}^{c}$ of the boundary feedback strategy (61) satisfies $k_{\rho}^{c}>v^{*}, 0<k_{v}^{c}<1$.

Remark 4: Our designed boundary feedback strategies (54) and (61) depend on the sign of the second velocity 

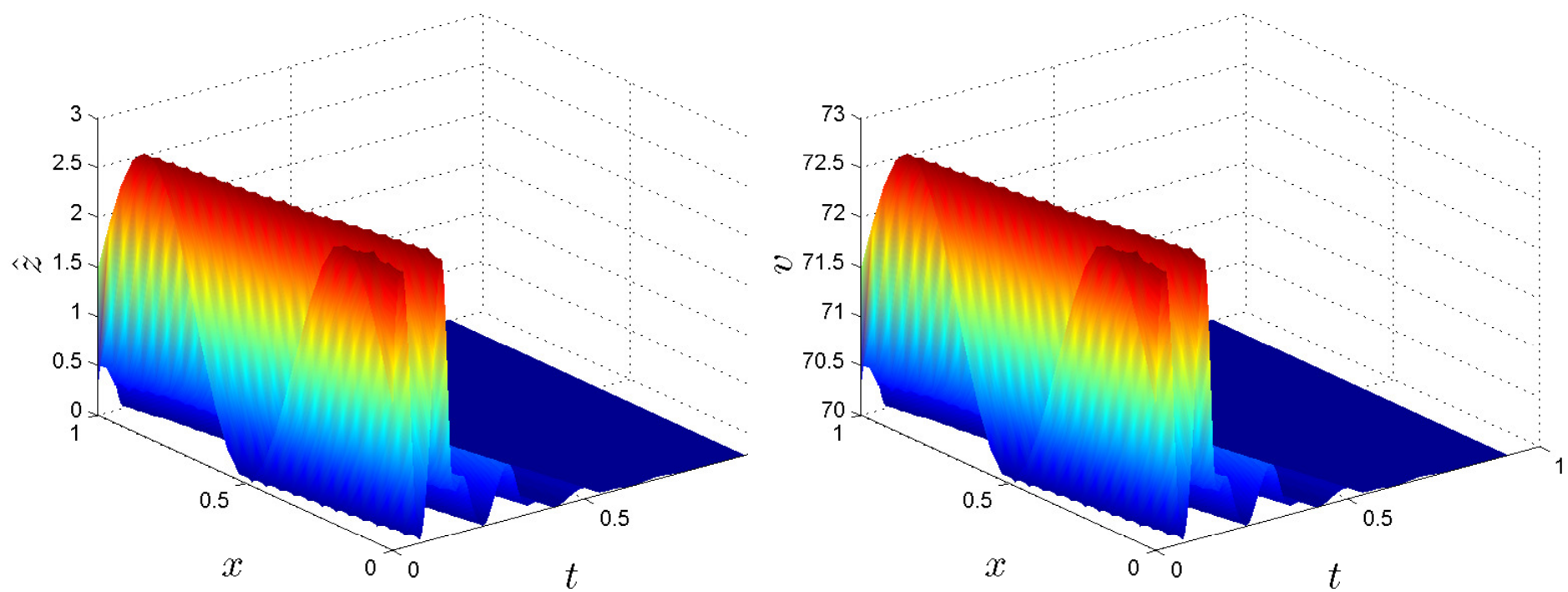

Fig. 7. The evolution of the second component $\hat{z}$ of the positive linear hyperbolic system (52) with matrix $G_{c}$.

Fig. 9. The evolution of average speed $v$ of the Aw-Rascle traffic flow equation (46) with the steady-state $\left(\rho_{2}^{*}, v_{2}^{*}\right)$.

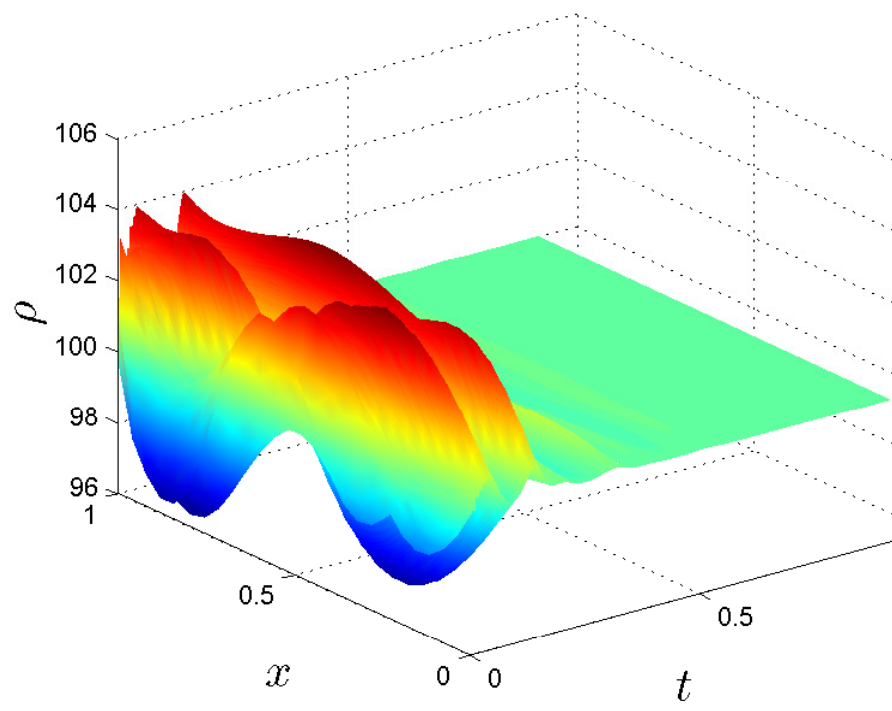

veh./hour, $p_{\text {out }}=8000$ veh./hour, and the total road length is $1 \mathrm{~km}$, i.e., $x \in[0,1]$.

Firstly, for the steady-state $\left(\rho_{1}^{*}, v_{1}^{*}\right)=(70,100)$, we have $\lambda_{2}^{*}=47.5>0$. In the neighborhood of this steady-state, the freeway traffic lies in the free-flow mode. Thus we apply the boundary feedback strategy (54), and the feedback gains are chosen as $k_{\rho}^{f}=20$, and $k_{v}^{f}=0.5$. The associated boundary condition matrix $G_{f}$ in (59) is given as

$$
G_{f}=\left[\begin{array}{cc}
0.2 & 0.0375 \\
0 & 0.5
\end{array}\right] \text {. }
$$

The initial deviations for the Aw-Rascle traffic flow equation (46)' are chosen as

$$
\left\{\begin{array}{l}
\rho(x, 0)=\rho^{*}+\sqrt{2} \sin (4 \pi x)+1.45 \\
v(x, 0)=v^{*}+\sqrt{2} \sin (3 \pi x)+1.45
\end{array} .\right.
$$

Figs. 2 and 3 show the time evolution of system (52) with

Fig. 8. The evolution of the density $\rho$ of the Aw-Rascle traffic flow equation (46) with the steady-state $\left(\rho_{2}^{*}, v_{2}^{*}\right)$.

which keeps either positive or negative in the whole spatial domain. For the spatial-varying steady-states containing phase transition between the free-flow mode and the congestion mode, the boundary feedback stabilization is not discussed in the present note.

\section{Simulation}

The developed boundary feedback strategies (54) and (61) are now tested with some numerical simulations in the two traffic modes presented above. To this end, we consider a local freeway section with parameters $\rho_{m}=200 \mathrm{veh} . / \mathrm{km}, v_{f}=150$ $\mathrm{km} /$ hour, $a=0.75, p_{\text {in }}=6000$ veh./hour, $r_{f}=r_{c}=1000$ the boundary feedback control (54), boundary condition matrix (59), and the initial conditions $\hat{w}(x, 0)=\hat{v}(x, 0)+a \hat{\rho}(x, 0)$, and $\hat{z}(x, 0)=\hat{v}(x, 0), x \in[0,1]$, under Riemann coordinates, satisfying the zero-order compatibility condition.

It is observed that solutions of $\hat{w}(x, t), \hat{z}(x, t)$ of hyperbolic system (52) preserve non-negativity and converge to the origin as time increases, as expected from Proposition 1 and Theorem 1. Figs. 4 and 5 show the solutions $\rho(x, t)$ and $v(x, t)$ to the Aw-Rascle equation (46). The states clearly converge to their steady-state $\rho_{1}^{*}=70$ veh. $/ \mathrm{km}$ and $v_{1}^{*}=100 \mathrm{~km} / \mathrm{hour}$, respectively, in these simulation.

In the other simulation, we assume freeway traffic lies in the congestion mode, and consider the steady-state as $\left(\rho_{2}^{*}, v_{2}^{*}\right)=(100,70)$. In this case, the second eigenvalue $\lambda_{2}^{*}=-5<0$. We apply the boundary feedback strategy (61), and the feedback gains are chosen as $k_{\rho}^{c}=400$ and $k_{v}^{c}=0.5$. 
Then the boundary condition matrix $G_{c}$ in (66) is calculated as

$$
G_{c}=\left[\begin{array}{cc}
0.175 & 1.0025 \\
0 & 0.2
\end{array}\right]
$$

Using the same initial conditions (69), Figs. 6 and 7 show that the boundary feedback control (61) stabilizes the linearized system (52). A common practice of traffic control during the congestion mode is to first regulate the downstream traffic to a constant value corresponding to the upstream measurements. Figs. 8 and 9 show that such strategy is effective to stabilize the congestion freeway traffic, by simulating the Aw-Rascle equation (46).

\section{CONCLUSiON}

In this paper, an important contribution was the necessary and sufficient conditions for the exponential stability of positive linear hyperbolic systems using a constructive linear Lyapunov function. This condition is written in terms of linear inequalities which are numerically tractable. The theoretical contribution was applied to stabilize freeway traffic represented by the Aw-Rascle traffic flow model. The control strategies combine the on-ramp metering with the speed limit in the distributed action.

Future work shall extend above theoretical results, such as Propositions 1, 2, and Theorem 1 with space-varying coefficients in the velocity matrix and with the discontinuous selection of boundary conditions. Some effort will also be devoted to the connection between the positivity of hyperbolic systems and the positivity of the associated Riemann coordinates.

\section{REFERENCES}

[1] S. Amin, F.M. Hante, and A.M. Bayen. Exponential stability of switched linear hyperbolic initial-boundary value problems. IEEE Transactions on Automatic Control, 57(2):291-301, 2012.

[2] A. Aw and M. Rascle. Resurrection of "second order" models of traffic flow. SIAM journal on applied mathematics, 60(3):916-938, 2000.

[3] G. Bastin and J.-M. Coron. On boundary feedback stabilization of nonuniform linear $2 \times 2$ hyperbolic systems over a bounded interval. Systems \& Control Letters, 60(11):190-196, 2011.

[4] G. Bastin and J.-M. Coron. Stability and Boundary Stabilization of 1-D Hyperbolic Systems. Progress in Nonlinear Differential Equations and Their Applications. Springer, 2016.

[5] J.-M. Coron, G. Bastin, and B. d'Andréa Novel. Dissipative boundary conditions for one-dimensional nonlinear hyperbolic systems. SIAM Journal on Control and Optimization, 47(3):1460-1498, 2008.

[6] J.-M. Coron, B. d'Andréa Novel, and G. Bastin. A strict Lyapunov function for boundary control of hyperbolic systems of conservation laws. IEEE Transactions on Automatic Control, 52(1):2-11, 2007.

[7] J. de Halleux, C. Prieur, J.-M. Coron, B. d'Andréa Novel, and G. Bastin. Boundary feedback control in networks of open channels. Automatica, 39:1365-1376, 2003.

[8] A. Diagne, G. Bastin, and J.-M. Coron. Lyapunov exponential stability of 1-D linear hyperbolic systems of balance laws. Automatica, 48(1):109-114, 2012.

[9] M. Dick, M. Gugat, and G. Leugering. Classical solutions and feedback stabilization for the gas flow in a sequence of pipes. Networks and Heterogeneous Media, 5(4):691-709, 2010.

[10] V. Dos Santos and C. Prieur. Boundary control of open channels with numerical and experimental validations. IEEE Transactions on Control Systems Technology, 16(6):1252-1264, 2008.

[11] P. W. Dower and P. M. Farrel. On linear control of backward pumped raman amplifiers. In IFAC Symposium on System Identification, pages 547-552, Newcastle, Australia, 2006.
[12] B. D. Greenshields. A study of traffic capacity. In Proceedings of the Highway Research Board, pages 448-477, 1935.

[13] M. Gugat, M. Dick, and G. Leugering. Gas flow in fan-shaped networks: classical solutions and feedback stabilization. SIAM Journal on Control and Optimization, 49(5):2101-2117, 2011.

[14] R. A. Horn and C. R. Johnson. Matrix analysis. Cambridge university press, 2012

[15] E. Kaszkurewicz and A. Bhaya. Matrix diagonal stability in systems and computation. Springer Science \& Business Media, 2012.

[16] B. S. Kerner. Introduction to modern traffic flow theory and control. Springer Berlin Heidelberg, 2009.

[17] T.-T. Li. Global classical solutions for quasilinear hyperbolic systems, volume 32 of RAM: Research in Applied Mathematics. Masson, Paris, 1994.

[18] F. Lorenzo and R. Sergio. Positive linear systems: theory and applications, volume 50. John Wiley \& Sons, 2011.

[19] O. Mason and R. N. Shorten. On linear copositive Lyapunov functions and the stability of switched positive linear systems. IEEE Transactions on Automatic Control, 52(7):1346-1349, 2007.

[20] M. Papageorgiou, C. Diakaki, V. Dinopoulou, A. Kotsialos, and Y. Wang. Review of road traffic control strategies. Proceedings of the IEEE, 91(12):2043-2067, 2003.

[21] C. Prieur, A. Girard, and E. Witrant. Stability of switched linear hyperbolic systems by Lyapunov techniques. IEEE Transactions on Automatic control, 59(8):2196-2202, 2014.

[22] C. Prieur and F. Mazenc. ISS-Lyapunov functions for time-varying hyperbolic systems of balance laws. Mathematics of Control, Signals, and Systems, 24(1):111-134, 2012.

[23] C. Prieur, J. Winkin, and G. Bastin. Robust boundary control of systems of conservation laws. Mathematics of Control, Signals, and Systems, 20(2):173-197, 2008.

[24] D. D. Siljak. Decentralized control of complex systems. Courier Corporation, 2011.

[25] M. Suzukiand and N. Sakamoto. Controlling ideal turbulence in timedelayed Chua's circuit: Stabilization and synchronization. International Journal of Bifurcation and Chaos, 20(05):1351-1363, 2010.

[26] H. M. Zhang. A non-equilibrium traffic model devoid of gas-like behavior. Transportation Research Part B, 36(3):275-290, 2002. 